(C) 2022, The Authors. Published by Elsevier Inc. and Fass Inc. on behalf of the American Dairy Science Association ${ }^{\circledR}$. This is an open access article under the CC BY license (http://creativecommons.org/licenses/by/4.0/).

\title{
Outer membrane protein A (OmpA) may be used as a novel target to enrich and detect Escherichia coli in milk samples
}

\author{
Yichen Tian, ${ }^{1,2} \odot$ Kaiqing Yang, ${ }^{2} \odot$ Yaoqiang Shi, ${ }^{1,2} \odot$ Jinyang Zhang, ${ }^{1,2} \odot$ Qinqin Han, ${ }^{1,2} \odot$ Xueshan Xia, ${ }^{1,2}$ \\ and Yuzhu Song ${ }^{1,2 *}$ (1) \\ ${ }^{1}$ Faculty of Life Science and Technology, Kunming University of Science and Technology, Kunming, 650500, Yunnan, China \\ ${ }^{2}$ Molecular Medicine Center of Yunnan Province, Kunming, 650500, Yunnan, China
}

\section{ABSTRACT}

In recent years, food safety incidents caused by Escherichia coli have occurred and have endangered human health. Due to the complex matrix of milk samples and the long pretreatment time, the existing methods cannot quickly detect $E$. coli in milk samples. It is necessary to enrich the $E$. coli in the complex matrix to improve the detection sensitivity. The E. coli outer membrane protein $\mathrm{A}(\mathrm{OmpA})$ is widely present on the cell membrane of $E$. coli and may be used as a new target to enrich $E$. coli. In this study, the purified recombinant OmpA protein was used to immunize BALB/c mice to produce polyclonal antibody. Immunomagnetic beads were combined with the polyclonal antibody to enrich the E. coli in the artificially contaminated milk samples. The products of immunoprecipitation were further used for PCR assay. The bacteria in the PCR sample can be pre-enriched, and the limit of detection is $10 \times 10^{0} \mathrm{cfu} / \mathrm{mL}$, which is about 100 times more sensitive than samples not processed by this method. Then, the artificially contaminated milk, coffee, juice, and soybean milk samples were tested separately, and it was found that the E. coli gene could be amplified. The whole analysis time was about 120 min, including the enrichment of bacteria and the detection of eluate. We found that OmpA combined with immunomagnetic beads was more efficient, fast, and convenient than the conventional method. Bacteria can be enriched more efficiently without extracting genomic DNA and culturing bacteria. Therefore, this method has potential value for improving the detection sensitivity and shortening the detection time of $E$. coli in food samples.

Key words: Escherichia coli, OmpA, milk, immunomagnetic bead, sensitivity

Received July 6, 2021

Accepted December 21, 2021

*Corresponding author: yuzhusong@kmust.edu.cn

\section{INTRODUCTION}

Escherichia coli bacteria are naturally found to colonize in the intestines of humans and various animals, but some special serotypes of $E$. coli can cause severe diarrhea and other adverse symptoms (Kim and Oh, 2020). Human E. coli infections can be roughly separated into intestinal and extra-intestinal infections. Oral intake of food contaminated with E. coli can cause intestinal infections, dehydration, and low blood pressure. Escherichia coli can cause human diseases by spreading through water and food. It can also bring risks to the society and economy due to global trade exchanges. Research on the detection methods of foodborne E. coli is a crucial link in dealing with food safety issues. Accurate and rapid foodborne E. coli detection technology must be studied to protect human health and reduce economic and trade losses (Wang et al., 2020; Bhardwaj et al., 2021). The development of rapid detection and control measures for $E$. coli have a great significance; the resolution and sensitivity of most rapid microbial detection technologies for microbial identification and counting are far from meeting the actual requirements. The limit of detection (LOD) for the colony-forming units in most foods in the food hygiene inspection standards is not very high. Moreover, the pre-processing of many detection methods are time-consuming and laborious, requiring multiple subcultures and biochemical tests (Park et al., 2020a). Therefore, a method is needed that can rapidly enrich $E$. coli in samples during the pretreatment stage. It can enrich E. coli in a limited concentration of samples, thereby avoiding pre-detecting culture time and also increasing sensitivity.

Outer membrane protein A (OmpA) is conserved in almost all E. coli (Smith et al., 2007; Confer and Ayalew, 2013), including commensal strains (Pocanschi et al., 2013). The OmpA protein is characterized by the N-terminal domain, which is composed of 8 transmembrane antiparallel $\beta$ barrel domains (Vogel and Jahnig, 1986; Nielsen et al., 2020). The 8-stranded chain is 


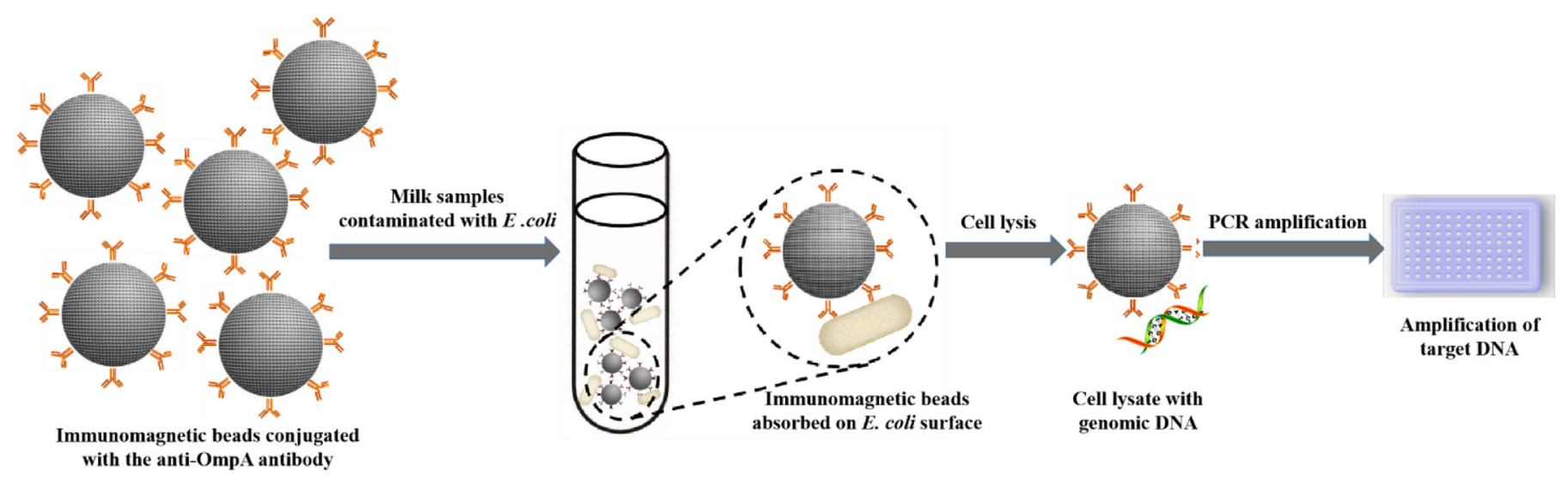

Figure 1. Flow chart of the detection method for Escherichia coli in milk samples using immunomagnetic separation combined with PCR.

formed by the periplasm of 4 long loops and 3 short corner folds on the outer membrane surface (Wang, 2002). Its main function is to maintain the integrity of the outer membrane (Khalid et al., 2008). The OmpA of $E$. coli is the most characteristic protein of OmpA family proteins (Confer and Ayalew, 2013). It is a hydrophilic protein with its immunogenicity related to the ring structure exposed on the surface of the molecule. It can be used as a target of the innate immune system to enhance the bactericidal effect or eliminate bacteria. Therefore, OmpA has been evaluated as useful rapid bacterial identification target to detect $E$. coli (Kese et al., 2011).

Immunomagnetic separation technology is a novel detection method to enrich bacteria, which can selectively capture the target bacteria to form the bead-bacteria compound. When an external magnetic field is applied, the complex can be assembled into a smaller volume to remove the complex matrix from the background. In this method, a specific polyclonal antibody is combined with magnetic beads to form immunomagnetic beads (IB). The IB adsorbs bacteria in the matrix (such as food) under the action of a magnetic field. Combined with molecular diagnostic technology (e.g., PCR), foodborne E. coli can be detected efficiently and accurately in milk samples (Park et al., 2020b).

In this study, we established a rapid and sensitive method to effectively enrich E. coli in a complex matrix environment such as liquid food samples. The IB combined with anti-OmpA specific antibody (IB-OmpA) can more efficiently enrich $E$. coli in liquid food samples. In combination with PCR technology, we attempted to establish and verify a diagnostic IB-OmpA-PCR based methodology for visual and real-time detection of the E. coli (Figure 1). According to fluorescence visualization and agarose gel electrophoresis, the IB-OmpA-
PCR can improve the detection sensitivity with LOD $10 \times 10^{0} \mathrm{cfu} / \mathrm{mL}$. This provides new ideas for the rapid diagnosis of common pathogens in clinical and food.

\section{MATERIALS AND METHODS}

\section{Materials}

The quality control strains and clinical strains used in this study included E. coli, Acinetobacter baumannii, Pseudomonas aeruginosa, Klebsiella pneumoniae, Shigella Castellani, and Staphylococcus aureus. Clinical samples were collected from patients at the First Affiliation Hospital of Kunming University of Science and Technology (Yunnan, China). Eighty clinical isolates (contained $20 \mathrm{E}$. coli isolates, $20 \mathrm{~A}$. baumannii isolates, $20 \mathrm{~K}$. pneumoniae isolates, and $20 \mathrm{P}$. aeruginosa isolates) were obtained from First Affiliation Hospital of Kunming University of Science and Technology. All strains were refreshed on Luria-Bertani liquid medium.

\section{Expression and Purification of OmpA}

The primers of nested PCR were used to amplify the ompA gene (Table 1A). The PCR product was recovered and connected with pMD19-T (Tiangen)

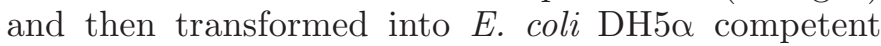
cells. The product and pET-32a (Tiangen) were enzyme-digested with Kpn I and Hind III (Tiangen), respectively. Then, the 2 fragments were connected and transformed into E. coli BL21 (DE3) competent cells. We used isopropyl- $\beta$-D-thiogalactopyranoside (IPTG; Sigma, Ronkonkoma) to induce the expression of OmpA. Nickel-nitrilotriacetic acid His·Bind Resin was used to purify the OmpA. 


\section{Preparation of Polyclonal Antibody Against OmpA}

Blood samples of clean mice were collected before immunization. The blood sample was incubated for $1 \mathrm{~h}$ at room temperature and then centrifuged at $3,913 \times g$ for 15 min at $4^{\circ} \mathrm{C}$. The serum was collected as a negative control. Mice were immunized by abdomen multipoint subcutaneous injection using $30 \mu \mathrm{g}$ of OmpA dissolved in $100 \mu \mathrm{L}$ of PBS, which was mixed with an equal volume of Freund's complete adjuvant (Gibco). After 2 wk, the Freund's complete adjuvant was replaced with Freund's incomplete adjuvant (Gibco) and the mice were immunized in the same way. A total of 4 immunizations were performed. After the third immunization, blood was taken from the tail vein for ELISA verification. The last immunization was a booster immunization, and $30 \mu \mathrm{g}$ of pure protein diluted with PBS was injected intraperitoneally. Subsequently, whole blood was collected $3 \mathrm{~d}$ later after the last immunization from the eye socket; the serum was collected according to the above described. The polyclonal antibody specific for OmpA was produced. We then added glycerin to the antibody at a ratio of 1:1, measured the concentration, and stored it at $-40^{\circ} \mathrm{C}$. Animal experiments and procedures were implemented in accordance with the guiding principles of animal experiments and approved by the Experimental Animal Committee of Kunming University of Science and Technology.

\section{Evaluation of Anti-OmpA Serum}

Serum titer was determined by ELISA. Different dilutions of anti-OmpA serum were used as an antibody for ELISA verification. Then $5 \mu \mathrm{g}$ of OmpA was diluted with PBS and coated on the wells of a microplate. The microplate was then incubated at $37^{\circ} \mathrm{C}$ for $3 \mathrm{~h}$. After block with the blocking buffer [5\% skim milk in PBS containing $0.05 \%$ Tween-20 (PBS-T)], the coated wells were incubated with $100 \mu \mathrm{L}$ of serum against OmpA with different dilutions (from 1:200 to 1:256,000) at $37^{\circ} \mathrm{C}$ for $2 \mathrm{~h}$. Then, we used goat anti-mouse $\operatorname{IgG}(\mathrm{H} \& \mathrm{~L})$ horseradish peroxidase (GenScript) as a secondary antibody for analysis. The optical density (OD) of each well was detected at $\mathrm{OD}_{450}$ using a microplate reader (Bio-Rad). Then, 80 clinical isolates were tested by ELISA to determine the cross-positive of serum, using clinical isolates as the antigen and anti-OmpA serum as the first antibody. The specific method is as above.

\section{Pretreatment of IB}

Fifty microliters of Enriching Beads Protein A (Enriching Biotechnology, MAg25K/Protein A) magnetic beads were transferred to the $1.5-\mathrm{mL}$ tube. The protein A magnetic beads were washed thoroughly with PBS-T. Then, magnetic separation was performed and washed 3 times by PBS-T. The anti-OmpA serum was diluted $(1: 2,000)$ into PBS-T. A total of $100 \mu \mathrm{L}$ of anti-OmpA serum was added into the $1.5-\mathrm{mL}$ tubes and mixed upside-down at $37^{\circ} \mathrm{C}$ for $15 \mathrm{~min}$. Magnetic separation was performed twice. The uncombined anti-OmpA serum were removed, then washed 3 times by PBS-T.

\section{The IB-OmpA-PCR Assay}

The IB were used to capture E. coli at $37^{\circ} \mathrm{C}$ for 20 min. Then, $20 \mu \mathrm{L}$ of elution buffer (100 $\mathrm{m} M$ Glycine, $\mathrm{pH} 2.8$ ) was added to the tubes and mixed gently with a pipette, to avoid bubbles, for $5 \mathrm{~min}$. Finally, $2 \mu \mathrm{L}$ of neutralization buffer ( $1 \mathrm{M}$ Tris- $\mathrm{HCl}, \mathrm{pH} 8.5)$ was added to neutralize the eluent. Then, $2 \mu \mathrm{L}$ of bacteria eluent was used as template for PCR amplification. Primers used for the PCR amplification reaction were hypothetical protein (GenBank ID: 13702648) previously designed by the laboratory (Table 1B; Li et al., 2019).

Table 1. Primers used in this article

\begin{tabular}{|c|c|c|}
\hline Primer $^{1}$ & Sequence $\left(5^{\prime}-3^{\prime}\right)$ & $\begin{array}{l}\text { Amplified } \\
\text { fragment } \\
\text { size (bp) }\end{array}$ \\
\hline \multicolumn{3}{|c|}{ Primer set A } \\
\hline F1 & 5'-ACGACAAGATGAAAAAGACAGCT-3' & 1.041 \\
\hline $\mathrm{F} 2$ & 5'-GGTACCGACGACGACGACAAGATGAA-3' & \\
\hline $\mathrm{R}$ & $5^{\prime}$-AAGCTTTTAAGCCTGCGGCT-3' & \\
\hline \multicolumn{3}{|c|}{ Primer set B ( Li et al., 2019) } \\
\hline F3 & 5'-ACCTCGCAACGTTGATTG-3' & 200 \\
\hline R3 & 5'-ACСТTCACCTTCCTTACG-3' & \\
\hline
\end{tabular}

${ }^{1}$ Primer set A is used to amplify the $o m p A$ gene by nested PCR. Primer set B is used to amplify the hypothetical protein gene. $\mathrm{F}=$ forward; $\mathrm{R}=$ reverse. 


\section{Detection of the Ability of IB-OmpA-PCR to Enrich E. coli}

The quality control strain of $E$. coli with a concentration of $10 \times 10^{3} \mathrm{cfu} / \mathrm{mL}$ was immunoadsorbed using IB that binds anti-OmpA antibody and IB that does not bind anti-OmpA antibody, respectively. We used PCR to verify whether or not the anti-OmpA antibody coupled with magnetic beads had the ability to enrich bacteria.

\section{Specificity and Sensitivity of IB-OmpA-PCR}

First, to check the LOD, the cultures of E. coli strains were serially diluted. Plate counting was performed 24 $\mathrm{h}$ after culture. Then, $100-\mu \mathrm{L}$ gradient dilutions with $E$. coli were inoculated into the milk samples with concentrations of E. coli ranging from $10 \times 10^{6}$ to $10 \times$ $10^{0} \mathrm{cfu} / \mathrm{mL}$. The samples were added to a $1.5-\mathrm{mL}$ tube, which contained magnetic beads-antibody compound, and incubated for $30 \mathrm{~min}$ at $37^{\circ} \mathrm{C}$. After incubation, the tubes were washed 3 times by PBS-T; then, 20 of $\mu \mathrm{L}$ elution buffer was added to the tubes, mixed gently with a pipette, to avoid bubbles, for 5 min to elute the bacteria. We added $2 \mu \mathrm{L}$ of neutralization buffer to neutralize the eluent. We used $2 \mu \mathrm{L}$ as a template and used the previously designed PCR primer for E. colispecific genes for IB-OmpA-PCR specificity detection. The sample without E. coli but with $K$. pneumoniae, Shigella, A. baumannii, S. aureus, or P. aeruginosa were used as negative controls to test specificity (Zhang et al., 2019).

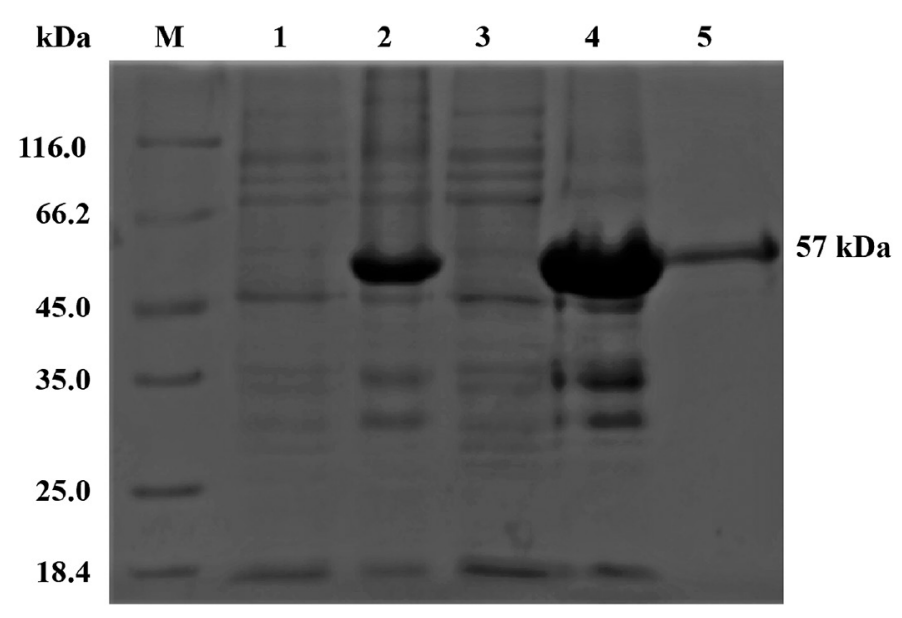

Figure 2. The SDS-PAGE analysis of expressed and purified OmpA. M: protein marker; lane 1: proteins before isopropyl- $\beta-D-$ thiogalactopyranoside (IPTG) induction; lane 2 : proteins after IPTG induction; lane 3: soluble fraction; lane 4: insoluble fraction; lane 5: OmpA purified by His.Bind Resin.

\section{Practical Application of IB-OmpA-PCR for the Detection of E. coli in Milk Samples}

To test the applicability of the IB-OmpA-PCR method in artificially inoculated milk samples (Zhang et al., 2018), a skim milk sample was purchased from a variety store and was identified as negative $E$. coli through traditional culture methods. The aliquots of $100-\mu \mathrm{L}$ gradient dilutions with $E$. coli were inoculated into the milk samples for final concentrations of $E$. coli of $10 \times 10^{6}$ to $10 \times 10^{0} \mathrm{cfu} / \mathrm{mL}$. Each milk sample was tested 3 times by IB-OmpA-PCR. We used the realtime PCR to test the sensitivity of liquid food samples (milk, coffee, juice, and soybean milk) to observe the level of sensitivity. The schematic diagram is shown in Figure 1.

\section{PCR Combined With Anthocyanin Visualization Experiment}

SYBR Green I is a commonly used nucleic acid dye. It can specifically bind to the double-stranded DNA double helix minor groove region, base interaction, and electrostatic interaction and emit fluorescent green fluorescence. Its fluorescence intensity becomes stronger as the concentration of the product in the sample increases; therefore, by observing the fluorescence intensity, we could roughly estimate the DNA content in the product. We diluted the SYBR Green I stock solution 1,000 times, added it in a ratio of 1:5, and mixed it. The fluorescence is generated soon after the addition and can be observed in the gel imaging system.

\section{RESULTS}

\section{Construction of Recombinant Plasmid and Purification of Protein}

The ompA gene was successfully cloned into the pET-32a cloning vector, and it was completely consistent with the sequence published by NCBI (https:// www.ncbi.nlm.nih.gov/). To verify the expression of the recombinant target protein, the host bacteria containing the recombinant plasmid were induced by IPTG to obtain a protein band with a molecular weight of about $57 \mathrm{kDa}$ in SDS-PAGE (Figure 2), including the OmpA protein about $37 \mathrm{kDa}$ and the $20.4-\mathrm{kDa}$ fusion protein tag of the pET-32a plasmid. This was consistent with expected size.

\section{Anti-OmpA Serum Can Bind Well to E. coli}

The reactivity of the anti-OmpA antibody was examined by ELISA, which was diluted in consecutive 
dilutions of 1:1,000 to 1:256,000. The titer of antibody was higher than 256,000 . This showed that the antibody titer reached the standard, and the follow-up test could be continued. The ELISA verification of 80 clinical strains showed that the anti-OmpA antibody had a strong response to E. coli (Figure 3). Therefore, this antibody can be used as a method for detecting $E$. coli.

\section{Detection of the Ability of IB-OmpA-PCR to Enrich E. coli}

It was found that the concentration of $E$. coli in the eluent using IB-OmpA-PCR was much higher than ordinary PCR, indicating that the anti-OmpA antibody did indeed adsorb E. coli and could enrich E. coli, thus increasing sensitivity (Figure 4).

\section{Specificity and Sensitivity of the IB-OmpA-PCR Assay}

In terms of specificity, none of the other 7 strains could amplify the E. coli gene (Figure $5 \mathrm{~A}$ ), in accord with previous findings that the specific genes against $E$. coli are specific and only respond to $E$. coli ( $\mathrm{Li}$ et al., 2019). The sensitivity of IB-OmpA-PCR was verified in gradient dilutions of $10 \times 10^{6}$ to $10 \times 10^{0} \mathrm{cfu} / \mathrm{mL}$. The results of the sensitivity test results showed that the LOD of IB-OmpA-PCR was up to $10 \times 10^{0} \mathrm{cfu} / \mathrm{mL}$ (Figure $5 \mathrm{~B}$ ).

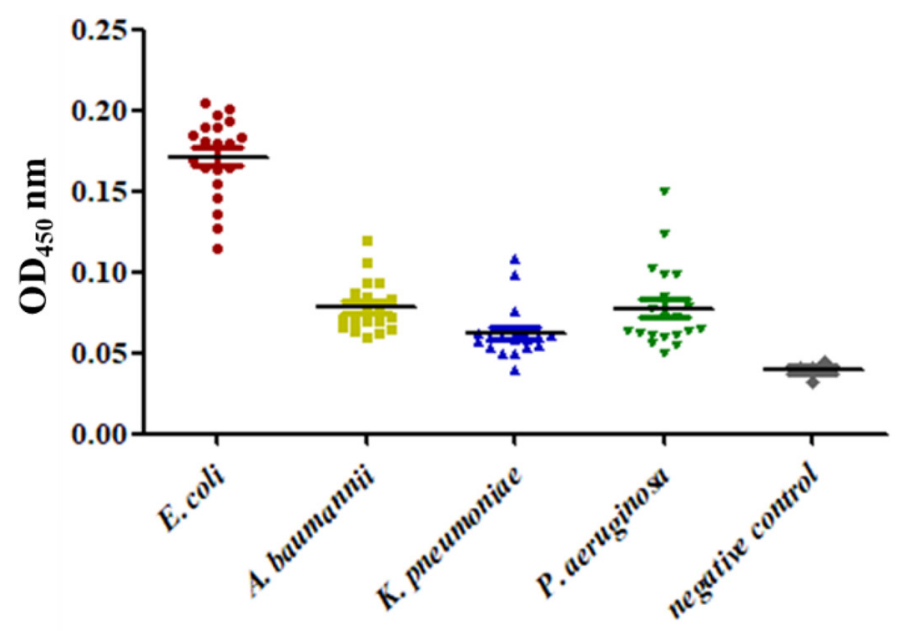

Clinical strains

Figure 3. The ELISA verification of clinical strains Escherichia coli, Acinetobacter baumannii, Klebsiella pneumoniae, and Pseudomonas aeruginosa. $\mathrm{OD}=$ optical density.

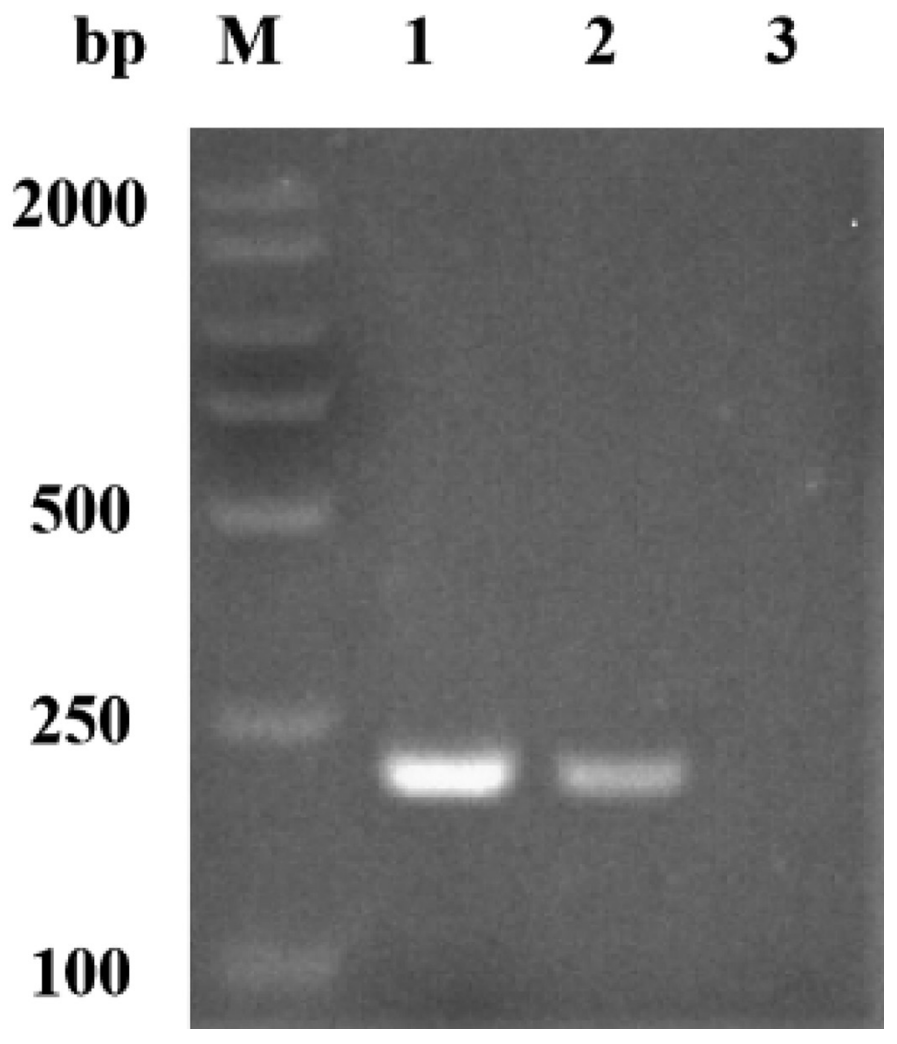

Figure 4. The concentration of $10 \times 10^{3} \mathrm{cfu} / \mathrm{mL}$ of Escherichia coli was enriched by IB-OmpA-PCR and PCR, respectively. Lane 1: IB-OmpA-PCR; lane 2: PCR; lane 3: nuclease-free water was used as a negative control. $\mathrm{IB}=$ immunomagnetic beads. $\mathrm{M}=\mathrm{DNA}$ marker DL2000.

\section{Practical Application of IB-OmpA-PCR for the Detection of E. coli in Food Samples}

The E. coli was serially diluted into milk samples (from $10 \times 10^{6}$ to $10 \times 10^{0} \mathrm{cfu} / \mathrm{mL}$ ) to test the sensitivity of using the IB-OmpA-PCR. The results showed that the green fluorescence and amplification bands were observed even at a concentration of $10 \times 10^{0} \mathrm{cfu} /$ $\mathrm{mL}$ (Figure $6 \mathrm{~A}$ ). On the contrary, the LOD of ordinary PCR is $10 \times 10^{2} \mathrm{cfu} / \mathrm{mL}$. The sensitivity of IB-OmpAPCR was about 100 times higher than that of ordinary PCR (Figure 6 B). Anti-OmpA antibody combined with magnetic beads can improve the sensitivity of detection. Using real-time PCR to detect different liquid food samples, it was found that $E$. coli could be detected (Figure 7) and the detection sensitivity was improved (data not shown).

\section{Clinical Utility of IB-OmpA-PCR for E. coli Detection}

The actual application of the IB-OmpA-PCR was verified using 22 clinical samples collected from the 
A

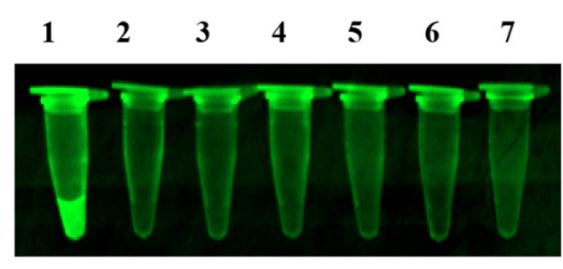

B

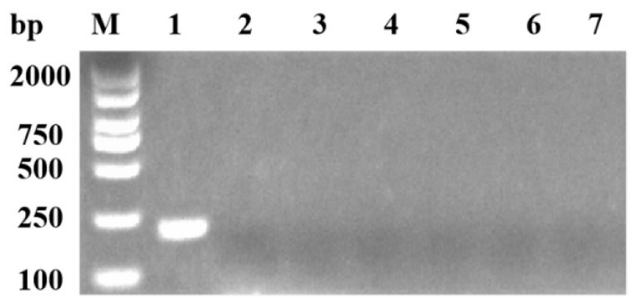

C

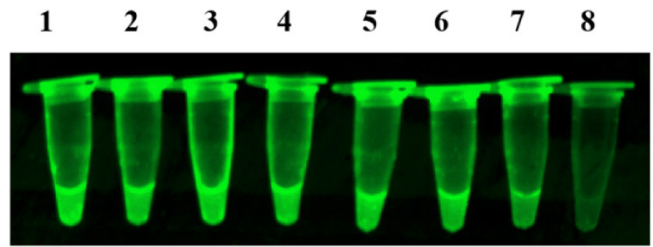

D

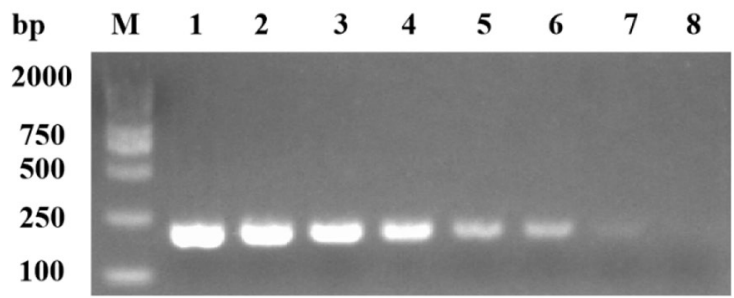

Figure 5. Specificity and sensitivity of the IB-OmpA-PCR method. (A) The fluorescence visualization of the IB-OmpA-PCR specificity reaction tubes. Tube 1 is the Escherichia coli eluent; the template in tubes 2 to 6 is the ordinal of the control strains, Klebsiella pneumonia, Shigella, Acinetobacter baumannii, Staphylococcus aureus, and Pseudomonas aeruginosa, whereas tube 7 is the negative control. (B) The agarose gel electrophoresis of (A). (C) The fluorescence visualization of the IB-OmpA-PCR sensitivity reaction tubes. Tubes 1 to 7 represent concentrations of $10 \times 10^{6}$ to $10 \times 10^{0} \mathrm{cfu} / \mathrm{mL}$ for milk samples eluted as template, respectively, and tube 8 is the negative control. (D) The agarose gel electrophoresis of $(\mathrm{C})$. IB = immunomagnetic beads. $\mathrm{M}=$ DNA marker DL2000.

hospital (Figure 8). Furthermore, 22 clinical samples were also identified by the traditional biochemical identification. These results are consistent with those of the IB-OmpA-PCR. The results demonstrated that the IB-OmpA-PCR was accurate, rapid, and specific for the detection of $E$. coli in clinical samples.

\section{DISCUSSION}

The rapid detection of foodborne pathogens is a key problem that needs to be solved urgently in the food industry. In particular, the detection of E. coli, has always been the top priority of food quality and

A

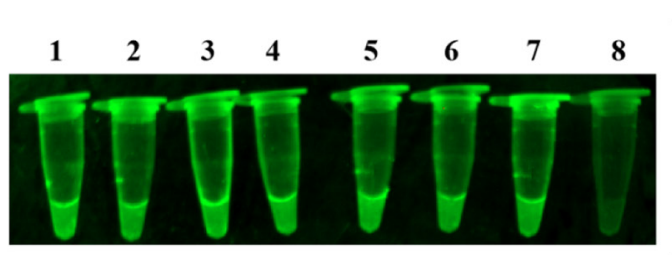

C

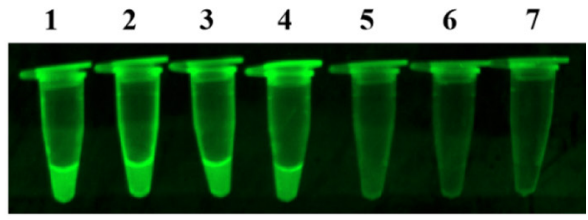

B

D
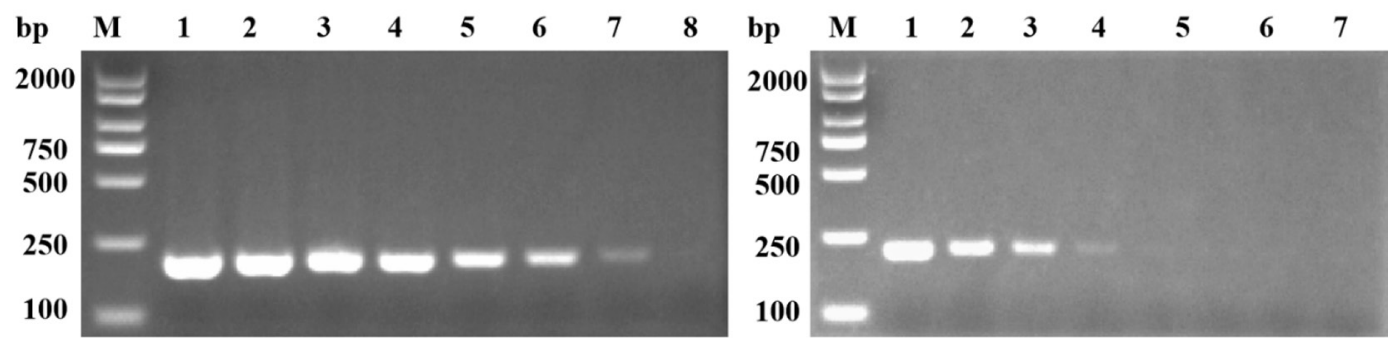

Figure 6. Comparison of the sensitivity between IB-OmpA-PCR and PCR. (A) The fluorescence visualization of the IB-OmpA-PCR sensitivity reaction tubes. Tubes 1 to 7 represent concentrations of $10 \times 10^{6}$ to $10 \times 10^{0} \mathrm{cfu} / \mathrm{mL}$ for milk samples eluted as template, respectively, and tube 8 is the negative control. (B) The agarose gel electrophoresis of (A). (C) The fluorescence visualization of the PCR sensitivity reaction tubes. Tubes 1 to 6 represent concentrations of $10 \times 10^{5}$ to $10 \times 10^{0} \mathrm{cfu} / \mathrm{mL}$ for milk samples eluted as template, respectively, and tube 7 is the negative control. (D) The agarose gel electrophoresis of (C). IB = immunomagnetic beads. M = DNA marker DL2000. 


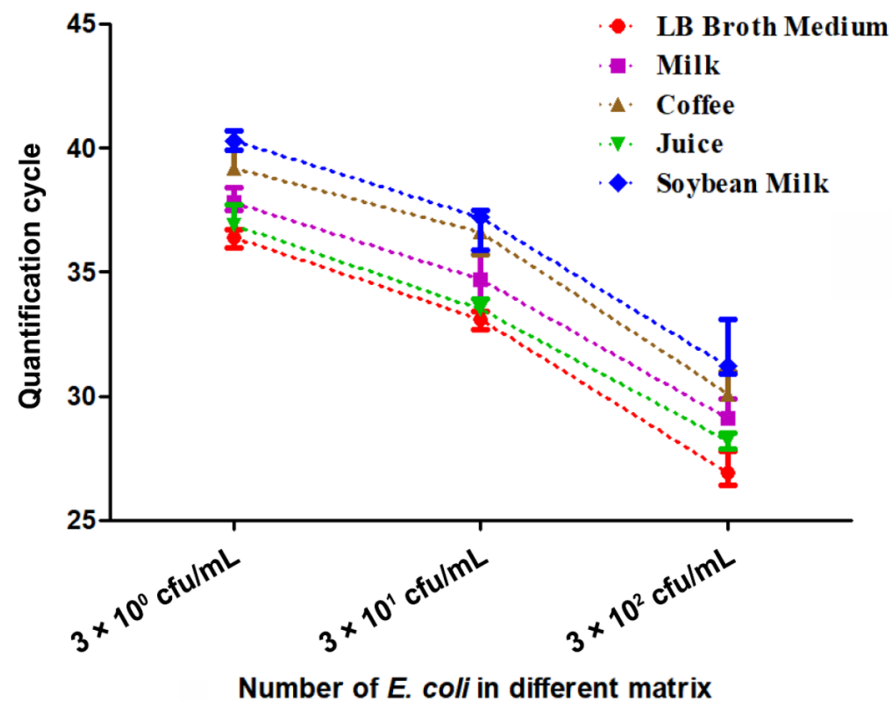

Figure 7. Detection of Escherichia coli in Luria-Bertani (LB) broth medium, milk, coffee, juice, and soybean milk using the IB-OmpAPCR and analysis of the eluted bacteria by real-time PCR. The average quantification cycle values are indicated with points, and the dotted line represents the standard curve. Error bars represent the standard deviation. $\mathrm{IB}=$ immunomagnetic beads.

safety research (Oliver et al., 2005). At present, the traditional detection methods of selective bacteria enrichment and biochemical identification are applied to the national detection standards. In the food hygiene testing standards, the LOD of most pathogens is $10^{3}$ to $10^{5} \mathrm{cfu} / \mathrm{mL}$, which cannot detect pathogens accurately and quickly. The identification resolution and sensitivity of rapid microbial detection technology needs to be further improved. With the rapid development of industry and economy, there is an increasing demand for rapid detection of pathogens, which greatly pro- motes the research and development of rapid detection technologies for various pathogens. In short, more rapid detection technologies will be practically applied and will replace traditional analysis methods to serve various detection departments.

Although the current stage of research on E. coli detection methods has been relatively in-depth ( $\mathrm{Li}$ et al., 2012), specificity and sensitivity are still major issues facing $E$. coli detection technology. It can be seen that there is still broad space for development in foodborne E. coli detection technology. Compared with the loop-mediated isothermal amplification, lateralflow strip immunoassays, immuno-gold nanoparticles, and conventional PCR, the IB-OmpA-PCR using antiOmpA antibody combined with IB can conveniently and rapidly enrich $E$. coli in liquid food samples. After enrichment, $E$. coli-specific PCR primers were used for amplification to obtain visible fluorescence for qualitative and quantitative analysis. The detection time was greatly shortened, and the entire detection time could efficiently detect $E$. coli in food samples within $120 \mathrm{~min}$ (Figure 9). As one of the important immunological detection methods, IB are widely used in the separation and detection of bacteria, which has been reported as an efficient method in isolating and concentrating target pathogens from complex matrices. Immunomagnetic separation has the advantages of high separation purity, high target activity retention rate, and high efficiency. It can be widely used in microorganism separation and purification, immunodetection, immunopurification, immunoprecipitation or other fields.

In addition, we did a detailed BLAST comparison (https://blast.ncbi.nlm.nih.gov/Blast.cgi) on the protein sequence of OmpA and found that it is widely present in gram-negative bacteria, and their OmpA

\section{A}

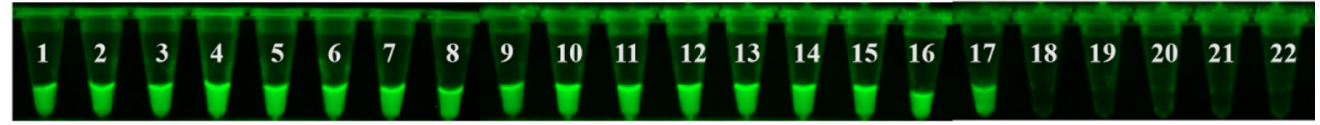

B

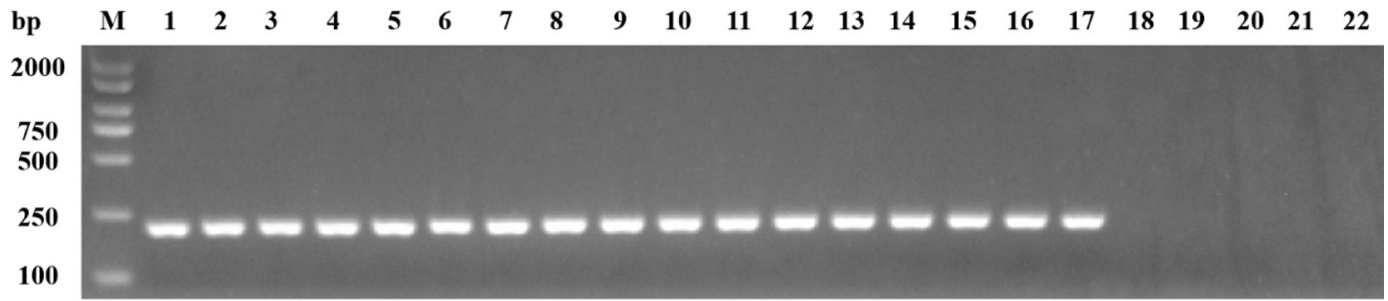

Figure 8. Detection of the 22 clinical isolates by IB-OmpA-PCR. (A) SYBR Green I was used to visually detect 17 positive clinical specimens and 5 negative clinical specimens. (B) Agarose gel electrophoresis was used to detect 17 positive clinical specimens and 5 negative clinical specimens. IB = immunomagnetic beads. $\mathrm{M}=\mathrm{DNA}$ marker DL2000. 


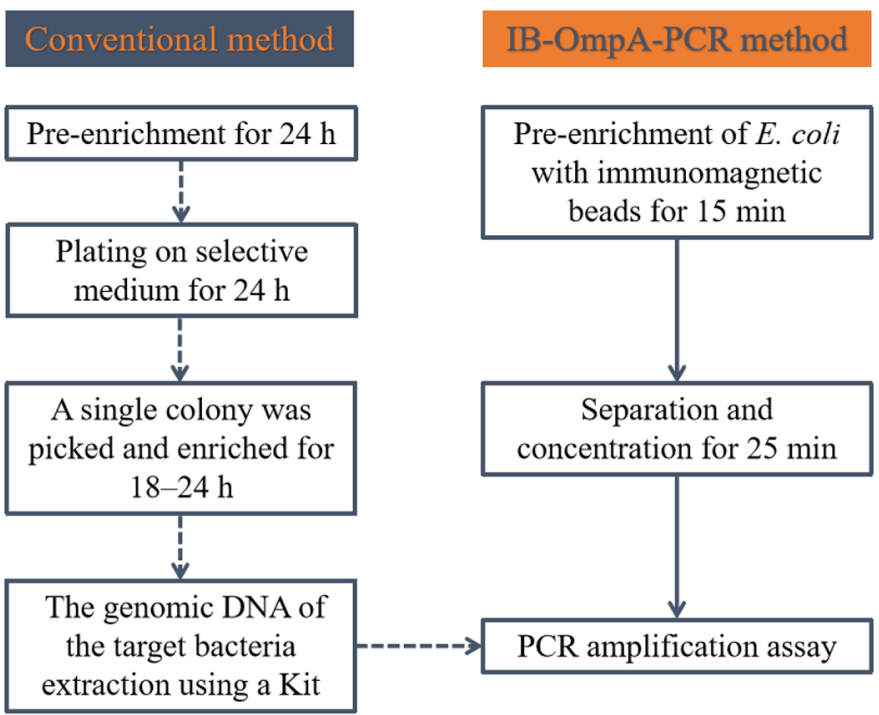

Figure 9. Procedure for the detection of target Escherichia coli in milk samples. Biochemical analysis of contaminated food samples was performed using conventional methods or the immunomagnetic separation pretreatment method proposed in this study. IB $=$ immunomagnetic beads.

protein sequences are extremely similar. It has a high degree of immunogenicity, which makes it have a wide range of applications. OmpA can be used as a target to enrich broad-spectrum gram-negative bacteria, or serve as potential targets for vaccine development. Previous studies in the laboratory found that anti-OmpA antibody is cross-positive to clinically common gramnegative bacteria to a certain extent (Figure 3). Figure 3 shows that OmpA is indeed widely present in clinically common gram-negative bacteria. In the future, we can try to study the linear epitopes of OmpA exposed outside the cell membrane to extract a small segment of common membrane protein specifically, so that a small segment of OmpA can be used as a targeted antibody to identify common clinical gram-negative bacteria. This antibody can identify a variety of common clinical bacteria, whether it is clinical testing or hemodialysis, and can greatly shorten the time of diagnosis and treatment.

\section{CONCLUSIONS}

A reliable method to monitor foodborne E. coli was established using IB-OmpA-PCR. This study verified the practicality of IB-OmpA-PCR for the enrichment of E. coli from artificially contaminated food. The target strain can be enriched rapidly in a complex matrix using anti-OmpA antibody. The operation is simple and takes only $120 \mathrm{~min}$, and its detection time and detection sensitivity are better than conventional detection methods. A convenient bacterial enrichment method and reliable PCR detection method allow IB-OmpAPCR to be applied for clinical and factory detection of $E$. coli. This method will lay a foundation for future immunomagnetic bead adsorption diagnostic methods.

\section{ACKNOWLEDGMENTS}

This work was supported by the Yunnan Provincial Department of Science and Technology grant number 2019ZF004-1, Yunnan, China]. Yichen Tian contributed to the conceptualization, methodology, and writing of the original draft. Kaiqing Yang and Yaoqiang Shi contributed to the data curation. Jinyang Zhang, Qinqin Han, and Xue-shan Xia contributed to the visualization, investigation, and project administration. Yuzhu Song contributed to the funding acquisition, writing, reviewing, and editing. Animal experiments have been approved by the local ethics committee. The work described was original research that has not been published previously, and not under consideration for publication elsewhere, in whole or in part. All the authors listed have approved the manuscript that is enclosed. The authors have not stated any conflicts of interest.

\section{REFERENCES}

Bhardwaj, D. K., N. K. Taneja, S. Dp, A. Chakotiya, P. Patel, P. Taneja, D. Sachdev, S. Gupta, and M. G. Sanal. 2021. Phenotypic and genotypic characterization of biofilm forming, antimicrobial resistant, pathogenic Escherichia coli isolated from Indian dairy and meat products. Int. J. Food Microbiol. 336:108899. https:// doi.org/10.1016/j.ijfoodmicro.2020.108899.

Confer, A. W., and S. Ayalew. 2013. The OmpA family of proteins: Roles in bacterial pathogenesis and immunity. Vet. Microbiol. 163:207-222. https://doi.org/10.1016/j.vetmic.2012.08.019.

Kese, D., M. Potocnik, M. Maticic, and R. Kogoj. 2011. Genotyping of Chlamydia trachomatis directly from urogenital and conjunctiva samples using an ompA gene pyrosequencing-based assay. FEMS Immunol. Med. Microbiol. 63:210-216. https://doi.org/10.1111/j .1574-695X.2011.00843.x.

Khalid, S., P. J. Bond, T. Carpenter, and M. S. Sansom. 2008. OmpA: Gating and dynamics via molecular dynamics simulations. Biochim. Biophys. Acta. 1778:1871-1880. https://doi.org/10.1016/j bbamem.2007.05.024.

Kim, J. H., and S. W. Oh. 2020. Rapid and sensitive detection of E. coli O157:H7 and S. Typhimurium in iceberg lettuce and cabbage using filtration, DNA concentration, and $\mathrm{qPCR}$ without enrichment. Food Chem. 327:127036. https://doi.org/10.1016/j .foodchem.2020.127036.

Li, C., Y. Q. Shi, G. Y. Yang, X. S. Xia, X. Q. Mao, Y. Fang, A. M. Zhang, and Y. Z. Song. 2019. Loop-mediated isothermal amplification assay for rapid detection of hypothetical protein gene in Escherichia coli clinical isolates. Clin. Lab. 65. https://doi.org/10 .7754/Clin.Lab.2018.180826.

Li, Y., P. Cheng, J. Gong, L. Fang, J. Deng, W. Liang, and J. Zheng. 2012. Amperometric immunosensor for the detection of Escherichia coli O157:H7 in food specimens. Anal. Biochem. 421:227-233. https://doi.org/10.1016/j.ab.2011.10.049. 
Nielsen, D. W., N. Ricker, N. L. Barbieri, H. K. Allen, L. K. Nolan, and C. M. Logue. 2020. Outer membrane protein A (OmpA) of extraintestinal pathogenic Escherichia coli. BMC Res. Notes 13:51. https://doi.org/10.1186/s13104-020-4917-5.

Oliver, S. P., B. M. Jayarao, and R. A. Almeida. 2005. Foodborne pathogens in milk and the dairy farm environment: food safety and public health implications. Foodborne Pathog. Dis. 2:115-129. https://doi.org/10.1089/fpd.2005.2.115.

Park, J. Y., M.-C. Lim, K. Park, G. Ok, H.-J. Chang, N. Lee, T. J. Park, and S.-W. Choi. 2020a. Detection of E. coli O157:H7 in food using automated immunomagnetic separation combined with real-time PCR. Processes (Basel) 8:908. https://doi.org/10.3390/ pr8080908.

Park, J. Y., K. Park, G. Ok, H. J. Chang, T. J. Park, S. W. Choi, and M. C. Lim. 2020b. Detection of Escherichia coli O157:H7 using automated immunomagnetic separation and enzyme-based colorimetric assay. Sensors (Basel) 20:1395. https://doi.org/10.3390/ s20051395.

Pocanschi, C. L., J. L. Popot, and J. H. Kleinschmidt. 2013. Folding and stability of outer membrane protein A (OmpA) from Escherichia coli in an amphipathic polymer, amphipol A8-35. Eur. Biophys. J. 42:103-118. https://doi.org/10.1007/s00249-013-0887-z.

Smith, S. G., V. Mahon, M. A. Lambert, and R. P. Fagan. 2007. A molecular Swiss army knife: OmpA structure, function and expression. FEMS Microbiol. Lett. 273:1-11. https://doi.org/10.1111/j .1574-6968.2007.00778.x.

Vogel, H., and F. Jahnig. 1986. Models for the structure of outer-membrane proteins of Escherichia coli derived from raman spectroscopy and prediction methods. J. Mol. Biol. 190:191-199. https://doi .org/10.1016/0022-2836(86)90292-5.
Wang, L., X. Shen, T. Wang, P. Chen, N. Qi, B. C. Yin, and B. C. Ye. 2020. A lateral flow strip combined with Cas9 nickase-triggered amplification reaction for dual food-borne pathogen detection. Biosens. Bioelectron. 165:112364. https://doi.org/10.1016/j.bios .2020.112364.

Wang, Y. 2002. The function of OmpA in Escherichia coli. Biochem. Biophys. Res. Commun. 292:396-401. https://doi.org/10.1006/ bbrc.2002.6657.

Zhang, L., Y. Shi, C. Chen, Q. Han, Q. Chen, X. Xia, Y. Song, and J. Zhang. 2019. Rapid, Visual Detection of Klebsiella pneumoniae using magnetic nanoparticles and a horseradish peroxidase-probe based immunosensor. J. Biomed. Nanotechnol. 15:1061-1071. https://doi.org/10.1166/jbn.2019.2736.

Zhang, L., Q. Wei, Q. Han, Q. Chen, W. Tai, J. Zhang, Y. Song, and X. Xia. 2018. Detection of Shigella in milk and clinical samples by magnetic immunocaptured-loop-mediated isothermal amplification assay. Front. Microbiol. 9:94. https://doi.org/10.3389/fmicb .2018 .00094 .

\section{ORCIDS}

Yichen Tian @ https://orcid.org/0000-0003-1773-226X

Kaiqing Yang @ https://orcid.org/0000-0002-6866-5565

Yaoqiang Shi $\odot$ https://orcid.org/0000-0001-6076-7711

Jinyang Zhang ๑ https://orcid.org/0000-0001-7533-998X

Qinqin Han (๑) https://orcid.org/0000-0001-9042-4170

Yuzhu Song (๑) https://orcid.org/0000-0002-9836-0252 